\title{
Nordiques
}

38 | 2019

Le petit héros scandinave

\section{Liliane Irlenbusch-Reynard, Rollon : de l'histoire à la fiction. État des sources et essai biographique}

Bruxelles, Berne, Berlin et al. (P.I.E. Peter Lang) 2016

Simon Lebouteiller

\section{OpenEdition}

\section{Journals}

Édition électronique

URL : http://journals.openedition.org/nordiques/302

DOI : 10.4000/nordiques.302

ISSN : 2777-8479

Éditeur :

Association Norden, Bibliothèque de Caen la mer

Édition imprimée

Date de publication : 1 novembre 2019

Pagination : 130-133

ISBN : 979-1-0959140-4-4

ISSN : $1761-7677$

\section{Référence électronique}

Simon Lebouteiller, « Liliane Irlenbusch-Reynard, Rollon : de l'histoire à la fiction. État des sources et essai biographique », Nordiques [En ligne], 38 | 2019, mis en ligne le 31 octobre 2020, consulté le 13 mars 2021. URL : http://journals.openedition.org/nordiques/302 ; DOI : https://doi.org/10.4000/nordiques. 302

Nordiques 


\section{Liliane Irlenbusch-Reynard} Rollon : de l'histoire à la fiction. État des sources et essai biographique

Peter Lang, 2016

Rollon est indéniablement un des personnages les plus importants de l'histoire de la Normandie et une figure majeure de la période viking. Actif dans la vallée de la Seine et les régions avoisinantes dès la fin du IX $^{\text {e }}$ siècle, c'est en effet ce chef viking qui conclut avec le roi carolingien Charles le Simple le fameux traité de Saint-Clair-sur-Epte de 911 qui permit l'établissement de Scandinaves dans la région et la création d'une entité territoriale et politique qui devint le duché de Normandie. Rollon fut alors le premier souverain de cette nouvelle principauté et le fondateur d'une dynastie ducale dont l'influence sur l'histoire de l'Occident médiéval fut majeure. En somme, la formation de la Normandie est une des entreprises des Scandinaves dont la postérité a été la plus notable.

Peu de Vikings comme Rollon ont été l'objet d'une littérature aussi abondante durant la période médiévale. On le retrouve ainsi dans les chroniques normandes qui le présentent à la fois comme un pirate païen ayant trouvé la rédemption et un duc glorieux, mais également dans les sagas islandaises qui font de lui un héros viking. Pourtant, Rollon reste de manière paradoxale un personnage particulièrement méconnu. Les sources à son sujet demeurent en effet dispersées, nous menant de la France à la Scandinavie, en passant par les îles Britanniques et même l'Italie. Les traditions sont par conséquent divergentes, voire contradictoires. Le caractère souvent tardif des textes et le problème de leur fiabilité compliquent également la reconstitution de son parcours. La tâche est d'autant plus difficile pour les chercheurs que ce travail nécessite une expertise sur des aires culturelles et des pratiques littéraires sensiblement différentes, de même que la mobilisation de compétences linguistiques diverses.

C'est justement à ces questions complexes que L. Irlenbusch-Reynard s'attaque dans le présent ouvrage, résultat d'un projet de recherche postdoctoral mené à l'université de Stavanger en Norvège. Elle propose de faire le point sur la documentation morcelée autour de Rollon et de récoler ces informations éparses. L'objectif est ainsi de reconstruire une biographie du premier prince de Normandie et de définir comment se sont formées les traditions historiographiques à son sujet. L'auteure réexamine les différentes hypothèses sur l'évolution de ce personnage et suggère quelques nouvelles pistes de réflexion. Il s'agit donc d'un travail de fond sur les sources qui accompagne utilement le livre publié la même année par Pierre Bouet, ce dernier faisant une présentation plus générale de la vie de Rollon et du cadre historique dans lequel il s'inscrivait ${ }^{1}$.

1 Pierre Bouet, Rollon : le chef viking qui fonda la Normandie, Paris, Tallandier, 2016. 
Le livre s'ouvre sur une introduction visant à restituer le contexte de l'installation de Rollon dans la vallée de la Seine, à travers le récit des premiers pillages en Francie, les causes du phénomène viking, la description des structures sociales et politiques en Scandinavie ou encore les conséquences de la conversion au christianisme et de la formation des monarchies nationales. La première partie de l'ouvrage, qui constitue à elle seule ses deux tiers, nous présente le corpus sur Rollon, région par région, en détaillant les renseignements que chaque document fournit. Les premiers textes mentionnant ce personnage ont été produits dans le monde franc, tels que les Annales et l'Histoire de l'Église de Reims de Flodoard. L'auteure relève ainsi des informations limitées et anecdotiques sur la création du duché et son fondateur, probablement parce que le sujet paraissait encore insignifiant aux yeux des chroniqueurs du $\mathrm{X}^{\mathrm{e}}$ siècle. Il faut donc attendre l'apparition d'une historiographie normande au début du $\mathrm{XI}^{\mathrm{e}}$ siècle avec l'important De moribus et actis primorum normanniae ducum de Dudon de Saint-Quentin pour obtenir les premiers récits substantiels sur la vie de Rollon. L. IrlenbuschReynard revient sur la fiabilité contestée de ce texte et les origines danoises que Dudon attribue à Rollon. La tradition initiée par le chroniqueur est ensuite poursuivie et adaptée par ses successeurs normands : Guillaume de Jumièges, Robert de Torigny, Wace, Benoît de Saint-Maure, etc. L'auteure se concentre ensuite sur les textes scandinaves, plus particulièrement islandais (Orkneyinga saga, Morkinskinna, Fagrskinna, Heimskringla, Knýtlinga saga...), qui développent au $\mathrm{XIII}^{\mathrm{e}}$ siècle une tradition spécifique et situent notamment les origines de Rollon dans la province norvégienne de Møre. Une nouvelle fois se pose la question de la fiabilité de ces récits tardifs qui sont avant tout le résultat d'une transmission orale de génération en génération et qui ont pu être l'objet de multiples altérations et inventions au fil du temps. On évoque ensuite les textes produits en Angleterre, ceux-ci étant largement redevables de l'œuvre de Dudon et de la documentation normande : Annales de Saint-Neots, Jean de Worcester, Guillaume de Malmesbury, Henry de Huntingdon, Siméon de Durham, Roger de Wendover... L'auteure mentionne enfin quelques témoignages isolés émanant de ces traditions, notamment au Pays de Galles et dans l'Italie normande.

Une fois ce tableau établi, l'auteure s'attelle à la difficile tâche de retracer les réseaux de circulation des informations sur la vie de Rollon. Elle suggère que l'œuvre de Dudon a voyagé tôt en Europe et a constitué le point de départ des différentes traditions formulées par la suite. Même en Scandinavie où des éléments locaux ont pu perdurer jusqu'à la composition des sagas, l'histoire de Rollon s'inscrit dans la continuité du chroniqueur normand selon l'auteure. Elle évoque ainsi les processus d'échanges de manuscrits 
et de connaissances qui ont dû s'opérer dès le $\mathrm{XI}^{\mathrm{e}}$ siècle, notamment par le biais des voyages des étudiants scandinaves sur le continent, le commerce ou encore les pèlerinages. Elle insiste alors sur le caractère composite de la production scandinave qui puise dans les traditions locales, normandes et anglaises et considère que les contacts entre Lincoln et Nidaros ont pu jouer un rôle moteur dans ces dynamiques.

Dans une dernière partie, l'auteure fait la synthèse des données sur la vie de Rollon. Une des nouveautés exposées dans l'ouvrage concerne l'origine contestée de ce personnage. Alors que les sources normandes suggèrent des racines danoises et les textes norrois des origines en Møre, les historiens actuels reprenant le plus souvent cette dernière hypothèse, L. IrlenbuschReynard se démarque en proposant que le père Rollon n'était pas le jarl Rögnvaldr de Møre, mais Rögnvaldr heðumhæri, un roitelet de Vestfold. Le chef viking serait ainsi venu du sud de la Norvège, région alors sous influence danoise, ce qui expliquerait son association avec la Dacie dans les sources normandes. L'auteure va même jusqu'à envisager que l'homme inhumé vers 900 dans la monumentale tombe à bateau de Gokstad puisse être le frère de Rollon lui-même. Pour ce qui est du parcours de ce dernier en Francie, l'auteure reprend ensuite les hypothèses communément admises par les historiens.
À plus d'un titre, l'ouvrage de L. Irlenbusch-Reynard est une belle contribution à l'étude des activités vikings en Normandie et des transferts littéraires vers la Scandinavie, d'autant plus que les publications sur ces sujets restent rares. On appréciera la qualité de l'expression qui rend la lecture agréable, malgré de nombreuses " coquilles " oubliées par l'éditeur. Un des apports majeurs du livre demeure la présentation systématique du vaste corpus sur Rollon. La description de chaque source et des informations qu'elles nous fournissent sera ainsi un outil particulièrement précieux pour les chercheurs et les étudiants travaillant sur les premières années du duché de Normandie et le phénomène viking.

Quelques idées formulées dans cette publication pourront toutefois être nuancées. L'hypothèse d'une origine de Rollon dans le Vestfold est intéressante et étayée par quelques indices, mais l'auteur ne parvient à démêler cette question épineuse. En l'occurrence, l'avis partagé par la plupart des historiens et déjà bien attesté dans les sources selon lequel il viendrait de Møre ne peut être écarté malgré ses faiblesses, d'autant plus qu'aucun texte ou élément concret n'indique clairement ses racines au sud de la Norvège. Selon les termes de l'auteure elle-même, cette proposition n'est qu'un "scénario possible " parmi d'autres. On pourra aussi discuter les procédés complexes d'échanges littéraires entre les régions qui ont mené à la formulation de traditions scandinaves. Si les transferts de 
l'Angleterre vers la Scandinavie, qu'il s'agisse de textes ou de récits oraux, paraissent indéniables, il demeure difficile, voire impossible, de reconstituer ces réseaux. Lauteure insiste pourtant sur le rôle d'un axe Lincoln-Nidaros par lequel aurait transité l'essentiel de la matière sur Rollon avant de passer en Islande. L'idée est pertinente, mais peut-être ne faut-il pas sous-estimer ici l'importance des contacts directs entre l'Islande et l'Angleterre, ou encore la capacité des Islandais à formuler leur propre tradition indépendamment de la Norvège. Les voyages de clercs islandais vers le sud sont en effet bien attestés et leur connaissance des textes circulant en Angleterre est mieux documentée qu'en Norvège. Même dans ce royaume, les auteurs islandais restaient ainsi des acteurs centraux de l'écriture de l'histoire et de la compilation des récits.

Ces remarques n'enlèvent cependant rien à la qualité générale de cet ouvrage que nous recommanderons aux lecteurs, chercheurs comme étudiants, souhaitant avoir une présentation complète de la vie de Rollon et des sources à son sujet.

Par Simon Lebouteiller 